\title{
Reflets
}

Revue ontaroise d'intervention sociale et communautaire

\section{Femmes et santé, en français s’il-vous-plaît}

\section{Cécile Coderre}

Volume 1, numéro 2, automne 1995

La santé communautaire en Ontario français : défis et espoirs

URI : https://id.erudit.org/iderudit/026076ar

DOI : https://doi.org/10.7202/026076ar

Aller au sommaire du numéro

Éditeur(s)

Reflets : Revue ontaroise d'intervention sociale et communautaire

ISSN

1203-4576 (imprimé)

1712-8498 (numérique)

Découvrir la revue

Citer cet article

Coderre, C. (1995). Femmes et santé, en français s'il-vous-plaît. Reflets, 1(2), 38-71. https://doi.org/10.7202/026076ar

\section{Résumé de l'article}

Les interventions des groupes de Franco-Ontariennes dans le champ de la santé remontent àla création même de ces groupes. Suite aux transformations qu'a connues la communautéfranco-ontarienne et à la présence accrue de l'intervention de l'État, les groupes de femmes audébut des années 80 ont interrogé le rapport des femmes à la santé. Ainsi, des services pourla communauté et la famille, leurs préoccupations se sont tournées vers elles-mêmes en créantdes formations et en revendiquant des services en français. Plus récemment, de nouvellessensibilités émergent; nouvelle définition de la santé, vision d'ensemble de la condition desFranco-Ontariennes et intégration des préoccupations des femmes des diverses communautésethniques.
Tous droits réservés (C) Reflets : Revue ontaroise d'intervention sociale et communautaire, 1995
Ce document est protégé par la loi sur le droit d'auteur. L'utilisation des services d'Érudit (y compris la reproduction) est assujettie à sa politique d'utilisation que vous pouvez consulter en ligne.

https://apropos.erudit.org/fr/usagers/politique-dutilisation/ 


\section{Femmes et santé,}

\section{en français s'il-vous-plaît}

L es interventions des groupes de $\mathrm{F}$ ranco- 0 ntariennes dans le champ de la santé remontent à la création même de ces groupes. Suite aux transformations qu'a connues la communauté franco-ontarienne et à la présence accoue de l'intervention de l'É tat, les groupes de femmes au début des années 80 ont interrogé le rapport des femmes à la santé. A insi, des services pour la communauté et la famille, leurs préoccupations se sont tournées vers elles-mêmes en créant des formations et en revendiquant des services en français. Plus récemment, de nouvelles sensibilités émergent; nouvelle définition de la santé, vision d'ensemble de la condition des F ranco-0 ntariennes et intégration des préoccupations des femmes des diverses communautés ethniques.

\section{Cécile Coderre}

É cole de service social, U niversité d' 0 ttawa

\section{Introduction}

L'apport important des groupes de femmes de l'0 ntario français à la recherche d'un mieux-être pour les femmes franco-ontariennes demeure peu connu malgré leur contribution significative à la promotion de la santé collective. N ous nous proposons de documenter cet apport par le biais d'une approche socio-historique. En plus des contributions des groupes de femmes, nous avons aussi présenté les initiatives et politiques gouvernementales ontariennes et fédérales touchant directement la santé des femmes, politiquesqui permettent d'éclairer certaines initiatives desgroupes de femmes.

Ayant eu recours à la recherche documentaire comme outil de collecte des données, nous avons voulu être aussi exhaustive que possible, tout en reconnaissant les limites imposées par la 
difficulté d'accès aux documents, brochures ou projets des différents groupes dans toutes les régions de l'O ntario. II est en fait périlleux de reconstituer l'histoire du mouvement de la santé des femmes en 0 ntario français car il englobe plusieurs activités, souvent ponctuelles, qui n'ont en commun que le domaine d'intérêt, domaine lui- même très vaste.

N ous avons divisé cet article en troistranches historiques correspondant aux trois étapes du développement du mouvement des femmes en 0 ntario français, soit la période du féminisme de la différence, celle du féminisme de l'égalité et celle de l'équité (C ardinal et Coderre, 1990). La première tranche regroupe les activités plus traditionnelles des groupes de femmes quant à leurs préoccupations face à leur santé. Centrés sur la famille et la communauté, les services qu' offrent les membres des associations féminines en font les protectrices de la santé. $M$ ais en même temps, c'est dans une valorisation du savoir-faire des mères que s'inscrivent le devoir et le droit de participer à la vie publique. $U$ ne seconde tranche historique est marquée par la renaissance du mouvement des femmes dans les années quatre-vingts. C'est dans le premier colloque Savoir c'est pouvoir, organisé par un regroupement de Franco-O ntariennes de l'Est en 1981, que nous avons repéré les prémisses de la première analyse féministe de la santé en $\mathrm{O}$ ntario français. Cette période se subdivise en deux moments clefs: une première période d'effervescence, autour du début de la décennie quatre-vingts, balisée sous le signe de la découverte et de la dénonciation, avec l'organisation de nombreuses activités publiques, notamment l'organisation de colloques, la naissance de groupes, l'offre de formations, une seconde tranche, après 1985, caractérisée par la volonté d'être entendues et s'inscrivant dans la multiplication des interventions de l'État dans les questions francophones et dans celles de la santé des femmes. U ne troisième période, se démarquant par sa recherche d'équité, est riche en ouvertures aux diverses communautésethnoculturelles de l'O ntario français, à une analyse féministe plus systématique des diverses composantes de la santé, perçue comme l'un des éléments de la réappropriation du corps des femmes et à l'émergence de revendications politiques plus provinciales. $M$ ais cette recherche 
«... les interventions des groupes de femmes franco-ontariennes ont été très intenses, se repérant entre autres par la création de réseaux de soutien et de formation pour les adivités de soins primaires et la mise en place de formations pour la prévention et la promotion de la santé mentale et de la santé en général. » d'équité se fait toujours dans l'esprit de faire reconnaître l'apport des femmes francophones au développement de leur communauté, d'où l'émergence d'une vision plus globale de l'équité pour les $F$ ranco- $O$ ntariennes.

À l'instar de Saillant (1991), nous concevons le thème de la santé sous deux angles. En premier lieu, il y a le rapport des femmes avec la santé et la maladie, en partant de la constatation que les femmes sont davantage que les hommes bénéficiaires des services de santé. Patientes ou clientes, elles sont l'objet «des formes de plus en plus sophistiquées de la médicalisation» (Guyon, dans Saillant, 1991:11). En deuxième lieu, elles sont aussi des actrices en tant que dispensatrices de soins de santé: «par les soins, elles contribuent directement à la production de la santé, incluant la promotion, la prévention, le maintien et le recouvrement» (Saillant, 1991:11). D ans ces deux axes du champ de la santé, les interventions des groupes de femmes franco-ontariennes ont été très intenses, se repérant entre autres par la création de réseaux de soutien et de formation pour les activités de soins primaires, et la mise en place de formations pour la prévention et la promotion de la santé mentale et de la santé en général. Leurs actions avaient en commun de privilégier une approche déprofessionnalisée de la santé. C ette approche, définie par R uzek (1978) et reprise par Saillant (1985), se caractérise par plusieurs composantes dont la démédicalisation, la réappropriation du corps, la mise en circulation de l'information et le développement des mécanismes d'intervention des usagères dans les lieux de décision (Saillant, 1985:749). C ependant, comme nous le verrons plus loin, seules quelques composantes de cette définition sont pertinentes pour analyser les interventions des groupes de femmes en 0 ntario français. 


\section{La santé et les services en français: une préoccupation ancienne dans les associations féminines}

«L es assodiations

féminines en 0 ntario veillaient à la valorisation des connaissances de même qu'au bienêtre moral de leurs membres. »
Parler de la santé, c'est évoquer d'emblée le rôle traditionnel assuré par les femmes dans la famille. Ce rôle se définit par la recherche du bien-être des membres de la famille, à travers I'alimentation et les soins aux proches et aux malades. C e travail se décompose en de nombreuses tâches non quantifiables, peu valorisées et quasi invisibles, «e travail profane de soins n'est au fond que l'un des aspects du travail domestique» (C usson, 1991:31). Et Graham (1984) d'ajouter que ce que font les parents pour leurs enfants est un travail pour la santé: création des conditionsfavorables à la bonne santé (chaleur, confort, propreté, nourriture, protection... ), soins aux malades, éducation à la santé, médiation avec l'extérieur et réponse appropriée aux urgences (Graham 1984, cité par C resson, 1991:31). Ces tâches ne furent pas toujours exécutées dans l'isolement du foyer ni dans la soumission. Les associations féminines en 0 ntario veillaient à la valorisation des connaissances de même qu'au bien-être moral de leurs membres. Accusées trop longtemps de n'avoir été que les courroies de transmission du pouvoir clérical, les associations féminines ont permis aux femmes de développer des compétences en organisation communautaire et en action politique, entre autres. C espremiersgroupesd'entraide avaient le souci de sortir lesfemmes de l'isolement, de leur offrir répit et soutien.

$D$ ans un document intitulé $L$ a part des femmes il faut la dire (1979) préparé par la Fédération des femmes canadiennesfrançaises (FFCF) à partir de 500 témoignages de femmes francophones vivant en milieu minoritaire, les femmes interviewées évoquent directement leurs préoccupations pour la santé des femmes et pour celle des membres de leur famille et plus largement, de leur implication communautaire. 
«L es femmes ont toujours trouvé le temps de s'occuper des pauvres, des malades, d'être sage femme ou enseignante, d'assurer le financement des paroisses par leurs ventes de charité, et ce, tout en menant des luttes scolaires ardues.》 (FFCF, 1979:2)

Ainsi, au-delà de la famille, les soins s'inscrivent aussi dans la communauté élargie (Lamoureux et Leseman, 1989).

Le féminisme de l'époque se définit par un féminisme de la différence, appelé aussi féminisme maternel. «I réfère à la conviction que le rôle spécifique de la femme en tant que mère lui impose le devoir et le droit de participer à la vie publique» (D umont, 1986:13). R eposant sur un constat des différences entre les rôles assumés par les femmes et les hommes, ses objectifs sont de valoriser et faire reconnaître la part des femmes. D ans le cas spécifique du champ de la santé, c'est à la fois de valoriser les fonctions de protectrices de la santé assumées par les femmes et à de mettre à contribution les compétences des femmes pour les besoins de la communauté élargie.

L'U nion catholique des fermières de la province de l'O ntario (UCFO), fondée en 1937, a été au coeur de la valorisation de ces tâches, de ce travail de prendre soin, assumé par les femmes. D evenue l'A ssociation des fermières de l'O ntario (AFo) en 1969, suite à une scission à l'intérieur de son membership, cette association regroupe des femmes et des jeunes filles des paroisses rurales de l'O ntario dont l'objectif de départ était de répondre aux besoins des femmes et de leur famille au lendemain de la crise économique des années 1930.

«E n plus de se perfectionner dans l'art aulinaire pour apprendre, entre autres à se servir de tout, les fermières apprenaient à tisser le lin, à se servir de la laine des moutons, à fabriquer des couvertures, des couvre pieds, des tapis, à faire des vêtements» (U F 0, 1987:28)

C ertains cercles décrivent ainsi leur activité dans les années quarante: on collectionnait des recettes, des trucs pour le nettoyage, le soin des plantes. Et si leurs compétences culinaires ont été 
utiles pour assurer le bien-être de leur famille, elles servent aussi au mieux-être de la collectivité. A insi, diverses activités de levées de fonds pour les oeuvres paroissiales ont été et sont toujours financées par la vente de produits culinaires préparés par leurs soins. N ous y voyons aussi une forme d'appropriation et de valorisation du savoir-faire des femmes.

U ne autre association, I'U nion culturelle des FrancoO ntariennes (u CFO) a été aussi très active en 0 ntario français et elle est aujourd'hui très présente dans le débat entourant la question de la santé des femmes. L'u c Fo poursuit des objectifs assez semblables à ceux de I'AFo dont elle est issue; quoique regroupant davantage de femmes des milieux urbains et ayant des visées plus marquées de promotion de l'égalité des Franco-O ntariennes, elle reste attachée à certaines valeurs religieuses. Les aspects artisanaux, les rencontres amicales entre membres sont une partie intégrante de la vie associative. Ses activités touchant la santé des femmes dans cette première tranche historique sont aussi liées à la valorisation de leur savoir-faire, à la promotion de l'entraide pour sortir de l'isolement ainsi qu'à l'organisation de services en français pour certaines populationstelles les personnes âgées (maintien à domicile), les personnes endeuillées (préparation de repas et soutien), les nouvelles mères (relevailles), les mères nécessiteuses (fabrication de vêtements). L'U cFo reconnaît et valorise aussi les connaissances des femmes dansle domaine des plantes médicales, des techniques non intrusives et des remèdes de grand-mères.

La plus ancienne des associations féminines en 0 ntario, la Fédération des femmes canadiennes-françaises de I'O ntario, a été fondée en 1914 dans le but «de contribuer aux oeuvres de la guerre; elle intercédait en faveur des démuni-e-s et défendait le fait français» (Brunet, C holette et Piché, 1988:17). C ette association regroupait quelques milliers de femmes francophones catholiques en $\mathrm{O}$ ntario et à travers le $\mathrm{C}$ anada (I bid:17). 0 rientée autour des paroisses, l'action de ses membres en 0 ntario était principalement axée vers des services bénévoles à la communauté. L'engagement de sa présidenteA ImandaW alker- $M$ archand envers les femmes et la protection de l'enfance est «une autre façon pour elle de manifester sa solidarité envers les femmes et 
«A vec le développement des soins de santé, le contrôle médical accu des divers moments de la vie gynécologique des femmes et la prise en charge d' une partie des services sodaux par l'É tat, les femmes francophones se voient partiaulièrement dépossédées des foncions qu'elles avaient assumées antérieurement. » d'appliquer la devise de la FFC F : P our nos foyers (B runet, 1992:112). L'Association se préoccupe de dossiers dans le domaine de la santé tels l'organisation de services de santé en français dans les petites communautés et le travail bénévole auprès des institutions hospitalières dans les centres urbains. Ainsi, dans les années soixante-dix, l'exécutif de la fFC de la section 0 ttawa- ouest cherche à regrouper des femmes de tous les âges pour mettre sur pied un ou des services susceptibles de répondre à des besoins urgents: rendre visite aux malades et aux personnes âgées, organiser des loisirs pour les jeunes et moins jeunes (artisanat, cours de recyclage, services d'orientation et de dépannage pour les fillesmères, les alcooliques (FFCF- $O$ uest, 1979). U ne demande de subvention au Secrétariat d'État avait pour titre un nom évocateur, $S^{\prime}$ aider tout en aidant les autres.

Avec le développement des soins de santé, le contrôle médical accru des divers moments de la vie gynécologique des femmes et la prise en charge d'une partie des services sociaux par l'État, les femmes francophones se voient particulièrement dépossédées des fonctions qu'elles avaient assumées antérieurement. C ependant, elles contribuent pleinement à cheminer vers une prise de conscience de leurs propres besoins, besoins en tant que femmes franco-phones. Ceci correspond à l'évolution du mouvement des femmes vers la revendication de l'égalité pour toutes. U ne partie des préoccupations sera reprise lors des travaux de la Commission Bird.

C e mouvement correspond aussi à d'importants changements dans la communauté franco- ontarienne, changements considérés par plus d'un comme des ruptures. « $₫$ a communauté francoontarienne doit accuser le choc d'une crise de civilisation, ce qui provoque une remise en question des valeurs traditionnelles tant sur le plan individuel que social» (C arrière, 1993:307). Les associations fémininesne sont pas étrangères à ces changements, voulant affirmer davantage leur identité sexuée au sein d'une communauté en changement. L'U c Fo se fait à ce titre représentative de cette nouvelle pensée: $\ll 0$ n entend maintenir notre principale oeuvre: les arts domestiques. Par contre, nous voulons 
d'abord intensifier notre travail auprès des jeunes (... ) et ensuite soutenir les causes qui nous touchent, les femmes» (C ôté, 1986:50).

\section{Revendiquer et créer des services de santé en français pour les Franco-Ontariennes}

Cette évolution des groupes de femmes franco-ontariennes est commune dans la francophonie et ailleurs au pays. Q ualifiée de féminisme de l'égalité, elle s'articule autour d'un questionnement sur les droits fondamentaux des femmes. Au nom de l'égalité, les femmes revendiquent le droit d'exercer toutes les professions (y compris le statut de collaboratrice), de participer aux différents pouvoirs et d'avoir accès aux droits civiques et civils. $D$ ans le cas des F ranco- 0 ntariennes, le droit à l'éducation en français et ce à tous les niveaux, de même que le droit à la justice en français ou encore à des services de santé en français colorent le débat et sont des constantes. Plus spécifiquement dans le champ de la santé, c'est au coeur des années soixante-dix et quatre-vingt qu'émergent les premières analyses féministes sur le contrôle du corps des femmes et la désappropriation de leur santé. De nombreux thèmes et sujets de débats touchent les rapports sociaux «mpliqués dans la médicalisation des différents cycles reliés à reproduction (contraception, maternité, ménopause), ainsi que les effets néfastes de l'interventionnisme biomédical sur la santé des femmes (Gaucher, Laurendeau et Trottier, 1981; Saillant, 1985). Alors qu'au cours de ces années, aux États-U nis et au Q uébec, de nombreux groupes de femmes créaient des centres de santé pour les femmes, un seul centre de ce type semble se dessiner dans l'Est ontarien. Le C entre d'auto-santé des femmes francophones d'O ttawa Vanier a pour but d'informer les femmes sur tous les aspects de la santé qui les concernent. L'existence de ce centre fut toutefois de courte durée. Par ailleurs, le thème de la santé des femmes sera porté par de nombreux groupes de 
femmes naissants. M ais ce sera dans une optique différente de celle de l'organisation de services directs et parallèles de santé que s'inscriront les actions des groupes de femmes de l'O ntario.

\section{De nouveaux groupes en effervescence et un changement dans les associations féminines}

D ans la mouvance des revendications pour l'égalité des femmes en 0 ccident, un ensemble d'événements marquent le mouvement naissant des femmes en 0 ntario français: création de nouveaux groupes et mutations des associations traditionnelles en particulier. Sans que l'on puisse identifier un mouvement spécifique pour la santé des femmes, on remarque que les actions des groupes de femmes questionnent le champ de la santé en dénonçant les injustices contre les femmes et la discrimination dont elles sont victimes.

Le groupe Pro-F emmes, présent à la fin des années soixantedix dans l'Est de la province, propose des activités d'animation auprès des Franco- $O$ ntariennes. $\mathrm{Ce}$ groupe est animé par des représentantes de plusieurs groupes de femmes franco-ontariennes dont la FFCF, I'U CFO, I'AFO. Intéressé par la mise sur pied de sessions de formation portant sur le travail de groupe, la planification de projets et d'activités, le développement personnel, ce groupe est aussi vivement préoccupé par la situation économique des femmes et particulièrement celle des femmes âgées. Si le dos sier de la santé n'est pas sa principale préoccupation, Pro-femmes dénonce néanmoinsl'inégalité et la discrimination dont sont victimes les femmes âgées.

Parmi les nouveaux groupes, mentionnons la collective ayant créé un outil d'information pour les 0 ntaroises intitulé L e tablier déposé et Le nouveau tablier déposé, journaux publiés entre août 1979 et 1981. Ce journal sera diffusé principalement dans l'Est ontarien avec des échos dans toute la francophonie, notamment 
«A utre élément du contexte général : au cours de la décennie soixante-dix, on assiste à une mutation des assodiations féminines dont le mandat s'élargit. » au M anitoba, au N ouveau-Brunswick, au Q uébec. U ne chronique santé apparaît dans le premier numéro mais elle n'est pas régulière. Le premier article porte sur l'hypertension (vol. 1, no 1) et le second sur l'obésité (vol. 1, no 2). D ans ces deux courts articles, les informations sont de type médical. Toutefois, le questionnement féministe sur les conditions socio-économicohistoriques à la base de ces problèmes de santé est absent. Le seul article portant sur une critique féministe de la gestion de la santé sera le compte rendu de la conférence de C olette Guillaumin à I'U niversité d'O ttawa sur la consommation différentielle entre hommes et femmes. La journaliste rapporte les propos de C olette Guillaumin au sujet des rapports de sexage présents dans la pratique de la médecine et des soins ainsi que des normes de beauté et d'esthétique pour les femmes (Laurendin, 1981:7-8). Plusieurs membres de cette collective font partie du comité organisateur du colloque Savoir c' est pouvoir et un numéro spécial sera consacré au colloque.

Autre élément du contexte général : au cours de la décennie soixante-dix, on assiste à une mutation des associations féminines dont le mandat s'élargit. Ainsi graduellement, l'AFo ouvre ses perspectivesà de nouveaux thèmes et en 1982, son congrès annuel est consacré à la santé des femmes. De plus, elle distribuera à ses membres de l'information au sujet du programme 0 dyssée, offert par l'u c Fo et s'adressant aux femmes vieillissantes. Enfin dans ses cours et ses conférences mensuelles, elle propose diverses activités de formation autour des questions de santé, d'hygiène ou d'alimentation. Au sein de l'u cFo, les années 80 sont marquées par la préparation et la création d'ateliers de formation touchant la prévention et la promotion de la santé. Ainsi, l'u c fo tient à informer ses membres de questions touchant la pré-ménopause, la nutrition, I'al coolisme et les tranquillisants mineurs. C ette association utilise, en plus de ses congrès annuels, ses formations et son journal L e C ommuniqué pour diffuser des informations sur la santé des femmes. Q uelques numéros spéciaux méritent d'être signalés: la santé mentale c'est œe qu'il vous faut... Parole de l'u c Fo, en mars 1992; les femmes et la santé mentale, en mars 1993. S'ajoutent indirectement à ce thème déjà large de la santé, les cours de 
«Q uoique ces groupes aient eu un intérêt pour la question de la santé, c'est dans le colloque Savoir c'est pouvoir, en mai 1981, que se dessine le début du questionnement féministe de la santé pour les $\mathrm{F}$ ranco0 ntariennes.» croissance personnelle, en particulier ceux d'affirmation de soi et de leadership, qui sont souvent des démarches de dévictimisation et de réappropriation de soi menant au mieux-être.

Les actions des groupes de femmes de l'O ntario ne sont pas vécues en vase clos, elless'alimentent des changements qui sont en cours au sein de la Fédération des femmes canadiennes françaises, organisme qui change de nom en 1985 pour devenir la Fédération nationale des femmescanadiennes françaises (FN FCF). La FN FCF prépare une vaste recherche, la première de ce genre sur la situation des femmes francophones hors Q uébec. Elle publiera un document intitulé $\mathrm{F}$ emmes et francophones: double infériorité. Lors des rencontres avec les femmes, I'auteure a identifié certains problèmes dont le manque d'information sur leurs droits, l'éducation des enfants, la santé et leur besoin de contact avec d'autres femmes francophones. Plus spécifiquement en 0 ntario, la Fédération des femmes canadiennes françaises 0 ntario (FFCF-O) établira ses nouvelles priorités aprèsêtre devenue une desassociations membres de la FNFCF.

En mai 1990, la FFCF-o inclura un volet d'échange collectif lors de son colloque annuel sur le thème $M$ ieux vaut prévenir que guérir. Ce premier questionnement sur la santé des femmes a permis de mettre à jour cet intérêt pour les membres de cette association. C'est pourquoi, dans leur recherche sur les conditions de vie des Franco- 0 ntariennes de 45 à 64 ans, elles ont introduit un volet santé (FFCF 0 ntario, 1992). La santé est abordée sous plusieurs angles: l'état de santé, les difficultés rencontrées lors de consultations auprès de professionnelles et de professionnels de la santé, l'usage d'alcool, le stress et la violence faite aux femmes et à leurs enfants.

Q uoique ces groupes aient eu un intérêt pour la question de la santé, c'est dans le colloque Savoir c'est pouvoir, en mai 1981, que se dessine le début du questionnement féministe de la santé pour les Franco- 0 ntariennes. Premier de ce genre dans l'Est ontarien et en 0 ntario français, ce colloque est le fruit d'une concertation entre les différentes associations féminines et les groupes féministes naissants. Sous l'égide du service de l'éducation 
communautaire du C ollège Algonquin et d'organismes communautaires, les organisatrices du colloque qui se veut un lieu d'information, de réflexion et d'action, profitent des activités liées du 8 mars pour réunir quelques 300 participantes provenant principalement de l'Est ontarien. Selon Francine D rouin (1981), qui en fait un compte rendu dans L e tablier déposé, ce colloque avait une double visée: d'une part une prise de conscience de la condition des Franco- $O$ ntariennes et d'autre part, l'identification de dossiers prioritaires pour les femmes de l'Est. En ce qui concerne la santé des femmes, les revendications les plus importantes touchent la présence de services spécialisés dans le domaine de la santé, la pratique de la sage-femmerie (légalisation de la profession et accès à leurs services à l'hôpital ou à domicile), et les soins aux femmes âgées (pensions de vieillesse et appui pour la mise en place d'un centre de jour au Centre Élisabeth-Bruyère pour les Francophones) (fonds FFCF-1). La pénurie de professionnels francophones de la santé est également dénoncée. Enfin, on revendique des services d'information et de documentation en français sur la santé des femmes et la mise sur pied, en collaboration avec les collèges communautaires et les groupes de femmes, de réunions, conférences et colloques d'information sur la santé des Franco-O ntariennes. La question des services de santé en français et le besoin d'information desfemmes dans ce domaine sont intrinsèquement liés. Par ailleurs, en revendiquant la mise sur pied de services de sages femmes, les participantes questionnent les rapports traditionnels entre femmes et médecins. Suite à ce colloque, une quinzaine de femmes se sont réunies pour assurer un suivi et neuf groupes de travail, dont un sur la santé, se sont constitués. Le regroupement des O ntaroises de l'Est se forme pour assurer la coordination de ces divers comités (H uot, 1981:8-9). D 'autres groupes de femmes naîtront dansla mouvance du questionnement soulevé lors du colloque dont le $\mathrm{C}$ entre d'accès pour femmes (CAF), créé à $\mathrm{O}$ ttawa en 1981.

D es activités subséquentes dans le cadre de la journée du 8 mars soulèveront aussi dansl'Est ontarien la question du rapport des femmes à la santé. Ainsi, en 1983, plus de 150 femmes se 
réunissent au $C$ entre $C$ arrefour d' $O$ ttawa pour célébrer la fête internationale des femmes ${ }^{1}$. C ertains ateliers traitent de travail, du pouvoir décisionnel, alors que d'autres sont orientés spécifiquement sur la santé. En effet, presque la moitié des ateliers portent sur des questions de santé, dans une perspective assez large: amour, amitié, sexualité; féminisme et sexualité; santé et maternité; santé et alimentation; passage de la vie (U n 8 mars ensemble, 1983b). II nous semble cependant que le thème de la santé demeure dans ces premières années une question parmi d'autres. Par contre, à la fin des années quatre- vingts, la question de la santé des femmes sera davantage intégrée lors de la création de nouveaux groupes. À ce titre, le groupe Féminin Pluri-E lles, formé en 1988 par les femmes de Thunder Bay et du N ordouest, cherche à créer un lieu de rencontre et à informer sur plusieurs thèmes, dont la santé des femmes, le retour aux études, les lois, la violence faite aux femmes (Scapinello, 1990:28).

Si les colloques annuels des associations provinciales font émerger périodiquement la question de la santé des femmes, un collectif, lui-même un des nouveaux groupes nés dans la mouvance du mouvement féministe d'égalité au sein de la communauté franco-ontarienne, s'est préoccupé de dresser un bilan de l'intervention féministe dans le $\mathrm{N}$ ord avec un volet important sur le thème de la santé (G arceau, 1992b)². A utour des questions de la santé, on y a traité des thèmes de l'épuisement professionnel (St-Pierre, 1992), de l'auto-santé (B rassard, 1992) et des nouvelles techniques de reproduction (Gauthier, 1992).

\section{La diffusion de connaissances pour favoriser une approche déprofessionnalisée de la santé}

Telle que définie par Saillant, l'approche déprofessionnalisée inclut la mise en circulation de l'information et la multiplication de ressources autres que médicales (Saillant, 1985:749). C'est à ces deux niveaux que les interventions des groupes de femmes 
«F ortes des connaissances féministes naissantes sur les différentes formes de désappropriation de la santé et du corps des femmes et sur le rôle de la sodalisation dans la production de la maladie mentale, les assodations féminines franco-ontariennes, particulièrement I'UCFO, ont créé de nombreuses formations pour faire connaître la problématique de la santé des femmes et outiller celles-ci pour se rédéfinir comme des actrices.» franco- ontariennes seront les plus intensives. Bien que les formations diffusées par les groupes de femmes ne puissent être qualifiées de thérapeutiques (elles ne le prétendaient d'ailleurs pas), elles sont constitutives d'une démarche de reprise en main de leur santé. Fortes des connaissances féministes naissantes sur les différentes formes de désappropriation de la santé et du corps des femmes et sur le rôle de la socialisation dans la production de la maladie mentale, les associations féminines franco-ontariennes, particulièrement l'u cFo, ont créé de nombreusesformations pour faire connaître la problématique de la santé desfemmes et outiller celles ci pour se rédéfinir comme des actrices. II nous semble toutefois qu'au sein de ces nombreuses initiatives, le thème de la santé mentale ait pris de l'ampleur, délaissant quelque peu d'autres thématiques. 0 utre les nombreuses formations qu'elle a distribuées dans toutes les régions, l'u cFo a préparé des colloques, rédigé des revues et bulletins et enfin profité d'une série télévisée produite parTV O ntario, É prises de santé.

Le programme $C$ 'est rien que vos nerfs est la première formation consacrée exclusivement à la question de la santé mentale des femmes en 0 ntario français. C réée en 1984, cette formation porte sur la consommation par les femmes de substances nocives comme les tranquillisants et la cigarette.

0 dyssée est quant à lui un programme d'information sur la ménopause. O riginaire du $\mathrm{Q}$ uébec et préparé par un regroupement féminin, les Dames de C hamplain, ce programme, comportant cinq ateliers rencontres, a pour but d'amener lesfemmes à se sentir responsables de leur santé. Somme toute, il offre une alternative à un système de santé contrôlé par les médecins, axé sur le curatif et s'attaquant principalement aux symptômes. En fait, cette alternative propose informations et partage tout en ayant comme finalité de donner aux femmes des moyens pour être en mesure de participer au processus de décision médicale $e^{3}$.

U n troisième programme, Stress au féminin ${ }^{4}$, est le premier conçu en 0 ntario par l'u cFo et adapté aux besoins des Franco0 ntariennes des milieux ruraux. Cette formation s'appuie sur les récentes analyses féministes quant à la santé des femmes, en 
évoquant d'emblée des préoccupations au sujet de la fréquentation plus grande des cliniques de santé mentale par les femmes, des hospitalisations importantes pour des problèmes mentaux et de l'utilisation alarmante de somnifères et de tranquillisants. Ce programme de prévention et de gestion du stress vise à aider les femmes à se prendre en main pour mieux contrôler leur santé physique et mentale. En fait, il s'agit d'apprendre à gérer son stress en connaissant le niveau de stress avec lequel chacune peut fonctionner optimalement (A dam, 1990a).

U n dernier programme de formation créé par l'ucfo à l'intention des Franco- 0 ntariennes, intitulé $L$ a santé apprivoisée, vise à sensibiliser les femmes à l'importance de la santé mentale. Son objectif principal est de favoriser le bien-être des participantes, de les renseigner sur la prévention des maladies mentales et d'assurer le développement, l'accessibilité et I'utilisation de ressources francophones en santé mentale. C ette formation propose aussi une série de six ateliers de trois heuresqui abordent la santé mentale, la connaissance de soi, la communication, la violence faite aux femmes; les deux derniers ateliers sont consacrés aux nouvelles approches de santé et comportent des éléments sur la santé holistique et la spiritualité. Se définissant comme programmes de prévention, $L$ a santé apprivoisée et $L$ e S tress au féminin, confirment la tradition de l'ucFo et des femmes membres des associations féminines francophones de l'O ntario dans leur souci de favoriser l'autonomie des femmes. De plus, dans la formation La santé apprivoisée, des liens se tissent clairement entre la santé mentale et la violence faite aux femmes. Alors que ces liens étaient déjà suggérés dans la vidéo La lumière des mots produite par l'u cFo et traitant de l'impact du dévoilement par lesfemmes victimes d'abus sexuel, cette dernière formation les nomme explicitement.

$O$ utre les formations offertes aux Franco- $O$ ntariennes de toutes les régions de l'O ntario, la diffusion de l'information constitue un autre aspect de l'intervention auprès des groupes de femmes caractérisée par l'approche déprofessionnalisée. À ce titre, F emmes d'action est une revue très précieuse pour informer les Franco0 ntariennes sur le thème de la santé. Q uel ques numéros ont été consacrés aux divers aspects de la santé des femmes, notamment 
N otre santé (avril 1987), Ê tre (juin-juillet-août 1990). Presque tous les numéros offrent des informations sur des services, des brochures ou dessuggestions de lectures touchant la santé des femmes. Tous les thèmes sont abordés, de la dénonciation du pouvoir médical (D elagrave, 1985) et des contraintes corporelles faites aux femmes par les régimes amaigrissants (A dam, 1989a), aux nouvelles techniques de reproduction (Brunet, 1994), en passant par une analyse politique des enjeux du champ de la santé pour les Franco-O ntariennes (Adam, 1992a). Plusieurs articles portant sur la santé mentale des femmes questionnent les diagnostics de maladie mentale assignés aux femmes de même que le double standard en matière de traitements psychologiques et psychiatriques (A dam 1989c:12). En liant le malaise individuel des femmes aux structures sociales dans lesquelles elles évoluent, les auteures des articles publiés dans $F$ emmes d'action proposent une analyse critique féministe qui met l'accent sur les rôles sexués et stéréotypés comme sources de ces malaises. Cette analyse se veut aussi un approfondissement de la spécificité d'être une femme vivant en contexte minoritaire.

«En plus de l'oppression psychologique envers les femmes, les femmes francophones qui vivent en milieu minoritaire, doivent affronter une autre forme de discrimination : I'isolement sodal et culturel. E lles perdent le sens d'appartenance à un groupe aulturel distind et sont confuses au niveau de leur identité personnelle. L es conséquences sont importantes sur l'estime de soi.» (A dam, 1989c14)

«L a santé des femmes est analysée à partir du contex te d'inégalité politique, sociale et économique marquant les rapports sociaux de sexes, »
Un autre outil de diffusion du savoir que développent les femmes a pris naissance àT $V O$ ntario qui a réalisé, conjointement avec le Collège G lendon, une série télévisée placée sous la responsabilité de Lorraine Gauthier. C ette série de treize émissions allie information, entrevues d'intervenantes de la communauté et informatrices-clefs. $\mathrm{O} n$ y offre un portrait global et une analyse politique de la santé des travailleuses à domicile; on traite d'images stéréotypées, de la sexualité, du contrôle de la maternité par les nouvelles techniques de reproduction. La santé des femmes est 
analysée à partir du contexte d'inégalité politique, sociale et économique marquant les rapports sociaux de sexes.

\section{Revendiquer les services en français}

Le Symposium de la femme francophone, le premier rassemblement des Franco- 0 ntariennes de toutes les régions, a été préparé de longue date par des représentantes de divers organismes de femmes. $C$ 'est le fruit d'une collaboration entre les associations féminines et les associations francophones, plus spécifiquement I'A ssociation canadienne-française de I'O ntario (ACFO). II rejoint deux mouvements, soit celui des femmes qui se cherche une plateforme politique provinciale et celui de la communauté francophone qui cherche à se doter d'un arsenal juridique essentiel à son développement.

C e Symposium s'est tenu àToronto les 15-16 octobre 1985. Pour les fins de ce colloque d'importance, le C onseil des affaires franco- ontariennes (CAFO) prépare un document-synthèse sur la situation des Franco- 0 ntariennes. D ivisé en sections correspondant aux différents ateliers, il comprend onze thèmes dont I'un porte sur la santé. Le bilan que le CAFo tire du rapport des femmes à la santé est plutôt alarmant. Ainsi, les femmes sont les plus grandes consommatrices de médicaments, elles souffrent davantage de dépression nerveuse et d'angoisse. De plus, il leur est difficile de choisir un médecin-gynécologue, surtout pour celles qui ne peuvent défrayer les coûts supplémentaires attribuables à leur éloignement des centres urbains. Le problème de l'accès à des services de santé en français est amplifié par la pénurie de professionnelles et professionnelsmédicaux et paramédicaux, en raison, notamment, de l'absence de programmes complets de formation en français. Les données du symposium soulignent aussi que les femmes âgées ont de multiples problèmes de santé et que

«... la femmefrancophone de plus de 64 ans en 0 ntario

n'a pas suffisamment d'appui psychologique et 
«... la question des services en français pour les femmes et plus généralement pour les $\mathrm{F}$ rancophones, prend id une couleur particulière puisque nous sommes à quelques mois du vote de la L oi 8 sur les services en français» » professionnel parce qu'il y a un manque d'établissements pour personnes âgées francophones, de centres médicaux bilingues et de personnel francophone pour le conseil et l'aide à demeure aux personnes âgées». (CAF0, 1985:25)

D ans les recommandations de l'atelier sur la santé, la question de l'accès aux services de santé prime sur celle des spécificités des services de santé pour les femmes.Toutefois, les participantes ont voulu souligner le manque de femmes professionnelles dans ce domaine. Elles rejoignent ainsi tout un ensemble de revendications du mouvement des femmes concernant l'accès des femmes à toutes les professions et la volonté des femmes d'être soignées par d'autres femmes.

Tout comme au colloque des 0 ntaroises de l'Est, la question des services en français pour les femmes et plus généralement pour les Francophones, prend ici une couleur particulière puis que nous sommes à quelques mois du vote de la Loi 8 sur les services en français. Les femmes ont ajouté leur voix collective à une communauté déjà active.

\section{La santé des femmes francophones, une question politique}

Comme nous l'avons souligné, lors de grands rassemblements (par exemple, le colloque Savoir c'est pouvoir, le Symposium sur la femme francophone), les F ranco- 0 ntariennes ont revendiqué des services en français dans le domaine de la santé. Par ailleurs, dans les formationsqu'elles ont développées, elles ont articulé des liens entre condition féminine, santé et spécificité du statut de minoritaire. Elles ont, par exemple, mis en relation perte de l'estime de soi et statut minoritaire. II nous semble essentiel d'affirmer que la question de la santé pour les Franco- 0 ntariennes ne peut se définir que dansle contexte de la double dynamique de femme 
et de francophone. D oublement opprimées (FNFCF, 1981), elles revendiquent le statut d'actrices dans les deux sphères: santé des femmes et revendications linguistiques et culturelles. Elles ont saisi l'occasion des débats sur la Loi 8 pour poursuivre leurs interventions visant le mieux-être des Franco-O ntariennes. A insi, après avoir assisté à la naissance d'une analyse féministe de la santé des femmes, à la création de services ayant une approche non médicalisée de la santé, les Franco- 0 ntariennes investissent un autre volet de l'action politique. Elles veulent définir, avec l'État, les priorités de santé pour les Franco-O ntariennes tout en cherchant une reconnaissance de leur spécificité. D eux moments leur donneront l'occasion de se définir. Le premier entoure les débats au sujet de la Loi 8 et le second touche les consultations publiques à propos des facteurs déterminants de la santé.

$R$ appelons que la Loi 8 sur les services en français est entrée en vigueur le 18 novembre 1989. Fruit de nombreuses pressions politiques, cette loi prévoit que des services publics en français seront offerts dans vingt- deux (22) régions désignées. U ne telle loi vient compléter d'autres acquis tels le statut officiel du français devant les tribunaux et dansle domaine de l'éducation (Welch, 1995). Toutefois, les différents organismes gouvernementaux avaient trois années pour se doter des structures et ressources nécessaires à l'implantation de services en français. Cette loi a eu des effets auprès des organismes gouvernementaux, effets que I'on pourrait qualifier d'opération de visibilité. Par contre, le développement de services de santé en français pour les femmes francophones est à peine amorcés.

L'opération de visibilité a pris plusieurs formes. Les organismes gouvernementaux et para-gouvernementaux spécifiquement préoccupés par la condition féminine (la Direction générale de la condition féminine et le C onseil consultatif de l'O ntario sur la condition féminine) ont adopté trois mesures complémentaires qui ont permis aux Franco- $O$ ntariennes de faire entendre leur voix: représentation de femmes francophones dans leur conseil d'administration, traduction de publications et documents d'études del'anglais au français et enfin inclusion de la question desfemmes francophones dans leurs avis ou politiques. 
D eux grandes consultations furent aussi organisées: tout d'abord, une série de consultations des groupes de femmes francophones par la Direction générale de la condition féminine de l'O ntario et le Conseil consultatif de l'O ntario sur la condition féminine et, par la suite, consultation des Francophones sur les déterminants de la santé. C es deux consultations font ressortir des préoccupations similaires chez les femmes: davantage de services en français, déprofessionnalisation des services et prise en compte des besoins et des expériences des femmes vivant en contexte minoritaire.

Ainsi, lors des consultations intitulées $D$ ial ogue femme à-femme, en novembre et décembre 1989, à T immins, H earst, Sudbury, W indsor, $\mathrm{O}$ ttawa et Toronto, les femmes francophones ont pu mieux connaître les priorités de la $D$ irection en matière de santé. Ces priorités sont de trois ordres: les nouvelles techniques de reproduction, la production de vidéos sur la sexualité des adolescentes et des adolescents et les conséquences du vieillissement sur les femmes.

Par ailleurs, suite au symposium pancanadien sur la santé des femmes en 1990, un G roupe de travail fédéral, provincial et territorial sur la santé des femmes (1990) est créé. Le rapport de ce groupe de travail donne d'abord un aperçu général des principaux problèmes de santé que rencontrent les femmes, puis en note les aspects économiques et sociaux liés à la vie desfemmes et évoque les rapports contradictoires entre les femmes et le système de soins de santé. L'année suivante, soit en 1991, le C onseil consultatif de l'O ntario sur la condition féminine présente un documentdébat sur les femmes et le vieillissement. En dix ans, c'est le deuxième rapport à se pencher sur leur situation. $M$ ais, fait nouveau, une section porte sur la situation des femmes âgées francophones. En effet, c'est sous la rubrique «es femmes plus âgées ayant des besoins spéciaux» que les femmes âgées francophones obtiennent droit de cité.

D eux éléments retiennent l'attention des membres du C onseil à propos des femmes francophones. I'accès aux services sociaux et de santé en français et leur situation économique (C onseil consultatif de l'O ntario sur la condition féminine, 1991a:25-26). U n autre document sur la santé mentale des femmes ontariennes, 
$\ll \ldots$ les centres de ressources communautaires, quoique préoccupés par la question de l'accès aux services en français, ne s'interrogent pas toujours sur la nature des soins prodigués et sur leur adaptation aux besoins des femmes » publié en 1990, arrivait à la même constatation: les femmes francophones sont identifiées parmi les femmes qui souffrent davantage d'isolement et de discrimination.

«A u cours de la consultation, nous avons rencontré de nombreuses femmes francophones Elles ont formulé un grand nombre de recommandations qui rappelaient le besoin de services en français et en anglais ${ }^{6}$. Les femmes francophones ont rarement accès à des services en français dans leurs collectivités. E lles doivent souvent se rendre à (sic) d'autres régions de la province où il est fréquent que leurs besoins linguistiques et culturels ne soient pas satisfaits » (C onseil consultatif de I'O ntario sur la condition féminine, 1991:12)

C'est une certaine forme de reconnaissance de la discrimination historique dont les Francophones ont été victimes en 0 ntario qui rend le Conseil consultatif, tout comme la Direction générale de la condition féminine, très sensibles à ces questions.

Par ailleurs, la création de services de santé pour les femmes en français reste en plan. Les services de santé en français, longtemps exigés par la population francophone, n'ont pas fait l'objet d'un débat féministe sur la question. A insi, les centres de ressources communautaires, quoique préoccupés par la question de l'accès aux services en français, ne sinterrogent pas toujours sur la nature des soins prodigués et sur leur adaptation aux besoins des femmes.

\section{Dialogue santé, les prémisses d'une analyse féministe de l'équité}

En 1987, le Conseil ontarien du premier ministre sur la santé (1987-1991) publie le document intitulé P rendre soin de la santé. Traitant des facteurs déterminants de la santé, ce document traite 
«... la santé en

0 ntario français se conjugue avec l'éducation, l'environnement, les services en français et la formation des professionnelles et des professionnels de la santé.» des facteurs sociaux, économiques et environnementaux qui influent sur la santé. Le C onseil considérait alors que «si l'on consacre trop de fonds au traitement des maladies, on pourrait compromettre la santé des 0 ntariens en réduisant les mesures visant à soutenir les facteurs déterminants fondamentaux de la santé» (C onseil du premier ministre sur la santé, le bien-être et la justice sociale, 1994:3). Suite à l'élection du gouvernement dirigé par les $\mathrm{N}$ éo-démocrates, le nouveau Conseil du Premier ministre sur la santé, le bien-être et la justice sociale ouvre de larges consultations publiques sur la santé. C'est dans le cadre de ces activités que les Franco- $O$ ntariennesse sont engagées par la voix deActionÉducation-Femmes 0 ntario (aÉF-O).

Action-Éducation-Femmes- 0 ntario s'est vu confier le mandat de dresser un portrait des facteurs déterminants en matière de santé au sein des collectivités francophones. Le point de vue qu'a adopté AÉF-O dans son approche des déterminants de la santé est féministe dans le sens où cet organisme prend en compte la réalité expérientielle des femmes, questionne la pertinence du modèle des déterminants de la santé et considère les personnes participantes à la consultation comme les expertes.

D e l'ensemble des recommandations issues des consultations se dégage une vision très large de la santé et des déterminants de la santé. En tout premier lieu, il apparait que la santé des femmes est liée à leur statut social et que de ce fait, toutes les politiques assurant l'équité des femmes sont essentielles à la santé des femmes (AÉF-0, 1993:73-74). D'autre part, la santé en 0 ntario français se conjugue avec l'éducation, l'environnement, les services en français et la formation des professionnelles et des professionnels de la santé. Sur ce dernier point, leurs recommandations rejoignent celles des participantes au Symposium de la femme franco-ontarienne de 1985. La formation des professionnelles et professionnels de la santé est à repenser, elle doit s'ouvrir aux femmes et tenir compte des besoins desfemmes dans les curriculum ${ }^{7}$.

II faut aussi noter que les recommandations font largement état du sexisme qui prévaut dans le système scolaire et dans le monde du travail et de la discrimination dont les femmes sont victimes (pauvreté différentielle àl'encontre desfemmes). En fait, 
la santé est une question d'éducation, d'accès à l'éducation, à l'activité physique, à un environnement sain, à un travail, en bref, à la justice sociale. $N$ ous voyons dans cette nouvelle thématique les prémisses d'une quête de l'équité. N ous définirons les revendications des groupes de femmes appartenant à un mouvement de recherche de l'équité dans le sens suivant: une vision globale du développement pour les femmes et l'articulation de la dynamique des services de santé en français conçus pour les femmes.

\section{Une vision globale de la santé des femmes}

Les féministes ont longtemps dénoncé l'impact de la violence sexuelle sur la santé mentale et physique des femmes. Lors du colloque Sensibiliser, D édider, A gir tenu à 0 ttawa les 14,15 et 16 octobre 1994, ces liens se sont clairement définis. Ce colloque, le premier consacré à la violence sexuelle et regroupant desfemmes de tout l'O ntario français, a manifestement pris le pari de jeter un regard global sur la violence et d'y inclure la question de la santé des femmes. En fait, c'est en dénonçant les abus et la violence faite aux femmes que l'on met en lumière leurs conséquences sur la santé des femmes. Par ailleurs, un nouveau thème apparaît dans les discussions. Alliant violence sexuelle et santé des femmes, la dénonciation des pratiques de mutilations sexuelles permettent aussi une rencontre, un partage et un échange entre toutes les femmes francophones de l'O ntario, sans distinction de leur origine ethnique.

D es trois ateliers consacrés à la question de la santé des femmes et leurs liens avec la violence, le premier porte sur la consommation de produits intoxiquants et ses liens avec les agressions à caractère sexuel. Le second est centré sur les causes physiques et psychologiques du dévoilement et ses conséquences sur la santé mentale et physique, et le troisième sur les causes et les symptômes liés à l'épuisement. U ne animation d'atelier porte sur la perception que les femmes ont des médecins et une autre sur la communi- 
«L es F ranco-

0 ntariennes soulignent aussi les difficultés supplémentaires de celles qui vivent dans les petits villages ou dans les régions rurales ou dans le $\mathrm{N}$ ord et questionnent les services de santé qui leur sont offerts. 》 cation entre les patientes et les médecins, ces activités visent à démystifier le rôle des professionnels de la santé et à donner des outils de prise en charge aux participantes.

Les actes du colloque reproduisent des témoignages de survivantes d'agressions sexuelles commises par des professionnels de la santé. Elles expriment leurs besoins en ces termes: formation d'enquêteuses d'expression française et appui financier lors des poursuites. D'autres témoignent de leur consommation d'alcool suite à des agressions sexuelles et de l'ignorance des médecins sur cette question; ains à chaque fois qu'elles le peuvent, les FrancoO ntariennes demandent que l'on forme les professionnelles et professionnels en français aux besoins spécifiques des femmes. $D$ 'autres enfin soulèvent que les médecins sont trop empressés à donner des médicaments:

«Trop souvent le médicament est la seule thérapie. $P$ rendre des médicaments, ça n' enlève pas le problème, ça fait juste le masquer.» (C olloque provincial sur les agressions à caradère sexuel, 1994:117)

Les Franco- 0 ntariennes soulignent aussi les difficultés supplémentaires de celles qui vivent dans les petits villages, dans les régions rurales, ou dans le $\mathrm{N}$ ord, et questionnent les services de santé qui leur sont offerts. Par exemple, à celles qui ont des besoins liés au contrôle de leur consommation de substances toxiques, il est nécessaire d'offrir des traitements orientés vers leurs besoins et de questionner les discours culpabilisants pour les femmes, particulièrement tout le discours de la co-dépendance. Elles voudraient qu'on leur assure l'accès aux thérapies alternatives et veulent développer des réseaux alternatifs incluant les intervenantes et les services. Enfin, elles constatent que les regroupements de femmes sont un lieu de ressourcement, $d$ 'entraide et de soutien essentiel. Pour éviter l'épuisement, ellesquestionnent le rôle traditionnel attribué aux femmes et proposent de trouver un nouvel équilibre entre donner et recevoir. O n assiste dans ce colloque à l'articulation de la problématique des services de santé conçus pour les femmes par des femmes. Toutefois, c'est 
"L e thème de

l'intégration des pratiques de mutilations sexuelles telles que définies par les femmes des différentes communautés où elles sont pratiqueés est porteur de cette nouvelle vision de la la sororité plurielle et pluraliste au sein des groupes de femmes franco-ontariennes.» par l'ajout d'une nouvelle question, celle des mutilations sexuelles, que les groupes de femmes Franco- 0 ntariennes développeront une vision globale du changement pour les femmes.

C ette vision globale n'est possible que par l'inclusion des différents mécanismes et fonctionnement des rapports sociaux de sexe dans une redéfinition des rapports ethicité-féminité ( $C$ ardinal, 1992). «Alors qu'elles s'affirment comme femmes et francophones, elles disent autrement qu'elles désirent participer à I'avènement d'une culture à la fois univer selle et plurielle capable d'intégrer le différences de sexes, de classes, d'ethnies, de régions; (C ardinal, 1992:22). Le thème de l'intégration des pratiques de mutilations sexuelles telles que définies par les femmes des différentes communautés où elles sont pratiqueés est porteur de cette nouvelle vision de la la sororité plurielle et pluraliste au sein des groupes de femmes franco- ontariennes.

À cet effet, le R éseau desfemmes du Sud a organisé, en 1994, un colloque portant justement sur les mutilations sexuelles. $\mathrm{Ce}$ groupe, créé en 1982, est le premier et le seul regroupement de femmes francophones de cette région (Godin, 1990:30). Ses premières activités portaient sur divers sujets d'actualité tels que la question constitutionnelle, l'autonomie financière et l'immigration. $M$ ais peu à peu, le groupe se spécialise sur les questions de la violence faite aux femmes, et crée, en collaboration avec le Collège G lendon, un centre de documentation sur la condition féminine. II fait également un travail de sensibilisation et de prévention dans les écoles. Son intérêt pour les questions des mutilations sexuelles provient de sa composition multiculturelle ains que de la naissance du R éseau des femmes noires francophones de Toronto métropolitain. C e colloque est le déclencheur d'une foule d'activités dans plusieurs régions de l'O ntario. Ainsi, lors du Colloque sur la violence sexuelle, il était acquis que le thème des mutilations sexuelles serait le catalyseur du questionnement entourant l'intégration des femmes des différentes communautés ethniques au sein de la francophonie ontarienne. De son côté, le $R$ éseau des intervenantes francophones sur la violence faite aux femmes dans la régions d' $\mathrm{O}$ ttawa- $\mathrm{C}$ arleton a aussi offert un atelier sur les mutilations sexuelles dans le cadre de ses journées de 
formation; la M aison d'Amitié, une maison de transition pour les femmes francophones d' $\mathrm{O}$ ttawa- $\mathrm{C}$ arleton a également offert de la formation sur ce sujet. Faisant le pont entre la violence faite aux femmes et la santé des femmes, le thème des mutilations sexuelles est aussi au coeur de la dénonciation par lesfemmes des rapports d'appropriation de leur corps.

\section{Conclusion}

L'analyse des pratiques d'intervention des groupes de femmes franco-ontariennes fait ressortir un intérêt marqué sinon crucial pour les questions touchant la santé desfemmes. À ce titre, il faut noter que selon une recherche auprès des femmes francophones vivant en contexte minoritaire, le thème de la santé englobait, en 1989, le plus grand nombre d'activités de formation offertes par les groupes de femmes francophones au C anada, à l'exclusion du Q uébec (C ardinal et Coderre, 1991:57). De plus, une première lecture de leurs interventions dans le domaine de la santé fait émerger des priorités telles que la sensibilisation et la prévention en matière de santé mentale, mais aussi des faiblesses, dont celle de la dépendance face aux organismes subventionnaires. L'intérêt des groupes pour la promotion de la santé et la prévention rejoint certes les préoccupations des femmes et il s'articule bien avec le mandat de ces associations, qui est de promouvoir l'égalité pour lesfemmes. C ependant, il est aussi marqué par des intérêts financiers. A insi, le champ de la promotion de la santé a été largement subventionné par le ministère de Santé et bien-être social $C$ anada, permettant ainsi aux associations féminines de diversifier leurs services aux membres. $C$ 'est ainsi que la formation M a santé apprivoisée, offerte principalement aux femmes de 55 ans et plus parce que l'organisme subventionnaire l'exigeait, n'a pu être poursuivie faute de subventions. Et pourtant, cette formation avait été couronnée de succès, et semblait bien répondre aux besoins des femmes franco-ontariennes. Par ailleurs, on 
«... l'avortement, le sida et l'homosexualité constituent des thématiques qui ne sont abordées que peu fréquemment et de façon fragmentée » constate que des thématiques ont été peu à peu délaissées telles, entre autres, la sage-femmerie, la sexualité et la santé des femmes âgées. Enfin, d'autres thématiques ont à peine émergé, al ors qu'elles sont au coeur du questionnement féministe. Ainsi, on remarque des silences éloquents et de grandes absences dans le débat sur la santé des femmes: I'avortement, le sida et l'homosexualité constituent des thématiques qui ne sont abordées que peu fréquemment et de façon fragmentée. Ainsi le R egroupement des O ntaroises de l'Est (ROE), dont le mandat avait été assez large depuis ses débuts, choisit à la fin des années 80 , de se spécialiser et d'orienter son action politique sur le libre choix en matière d'avortement (Paquette et Lalande, 1990). Ce groupe sera I'un des seuls regroupements francophones à se pencher sur cette option puisque l'affiliation religieuse de plusieurs associations féminines colore les débats sur l'avortement. C e n'est que tardivement qu'un premier article dans la presse féminine francophone en milieu minoritaire porte sur le sida. En mars 1993, la revue F emmes d'action donne la parole à une animatrice du Comité E.L.L.E.S. d'O ttawa, qui tente de sortir du silence la question du sida chez les femmes. L'auteure souligne que les femmes sont encore responsables de leur protection et que paradoxalement, elles se perçoivent trop souvent comme immunisées, parce que peu conscientisées ou informées des risques. Les femmes porteuses du virus ou sidéennes sont seules, rejetées, sans service spécialisé. La sexualité a connu un traitement différent de celui de l'avortement. II serait faux de dire qu'on ignore ce sujet puisque divers ateliers étaient organisés sur ce thème lors des célébrations du 8 mars. $D$ 'autre part, ce thème a été très largement évoqué, mais en creux, lors des actions contre la pornographie. En effet, les Franco- 0 ntariennes ont été très actives aux moments forts de la lutte contre la pornographie au $\mathrm{C}$ anada, mais si elles dénonçaient cette forme d'appropriation desfemmes, elles n'ont pas défini leur position sur la sexualité. Ce n'est éga lement que tardivement que l'orientation sexuelle a été nommée.

Enfin, contrairement à la thématique de la violence qui regroupe quantité de groupes au sein de la coalition A cion ontarienne contre la violence faite aux femmes, la santé des femmes en 0 ntario 
«L e financement poncuel et non récurrent des programmes de formation ne permet pas de faire une analyse politique des enjeux de santé pour les femmes francoontariennes, ni encore d'établir un plan d'ađion, mêmeà moyen terme.» français n'a pas donné naissance à une coalition. Des raisons financières expliquent partiellement cette situation. En effet, les organismes subventionnaires précédemment cités financent principalement des programmes de prévention et de sensibilisation. Le financement ponctuel et non récurrent des programmes de formation ne permet pas de faire une analyse politique des enjeux de santé pour les femmes franco-ontariennes, ni encore d'établir un plan d'action, même à moyen terme. $U \mathrm{n}$ tel financement exige en fait de changer de perspectives, et de s'ouvrir à de nouveaux thèmes tous les trois ans. À ce titre, les groupes de femmes sont à la remorque des organismes subventionnaires. II faut enfin ajouter que les femmes franco-ontariennes n'ont pas clairement manifesté leur volonté de créer une coalition sur le thème de la santé. Lorsqu'il a été nécessaire de se regrouper, comme sur la question des nouvelles technologies de reproduction, une coalition temporaire s'est formée. C ette coalition à temps partiel est le fruit du travail de collaboration et d'échanges constants entre groupes. Toutefois, pour se définir comme des partenaires, il faut avoir plusieurs clefs en main, et actuellement, c'est le gouvernement qui en contrôle pluseurs. Ceslimitesfinancièresn'ont cependant pas paral ysé les groupes de femmes; le partenariat avec l'É tat constitue toujours une préoccupation majeure de leurs interventions. $D$ ans cet optique, la Table féministe de l'O ntario a un comité santé qui a le mandat d'articuler les revendications politiques des Franco- 0 ntariennes.

Il apparaît néanmoins que mal gré le peu de services, les avancées sont importantes. Les revendications se sont davantage affirmées, l'analyse féministe est omniprésente et elle sait se redéfinir au fil des années, au contact des formatrices, des membres, des groupes et des intervenantes. Ainsi, la perspective familialiste de la santé a peu à peu été délaissée en faveur de celle centrée sur l'analyse des rapports sociaux de sexe. Enfin, les femmes ont su se définir des formations à leur mesure et à leur image.

\section{Notes}


1. Les célébrations entourant le 8 mars ont été l'occasion en 0 ntario français de favoriser la concertation entre les groupes. Ainsi en 1983, le thème du 8 mars est U n 8 mars ensemble et le comité organisateur se compose de membres de plusieurs organismes francophones: I'A ssociation des fermières de I'O ntario, Budget-Aide de $\mathrm{H}$ aw kesbury, Comité de promotion féminine de AEFO, C omité support à la famille, Fédération des femmes canadiennes- françaises, section $C$ asselman, Femmes-chefs de foyer de Casselman, $U$ nion culturelle des Franco-O ntariennes (U n 8 mars ensemble 1983:32) Fonds FFCF C 53\52 6 A ctivités, projet pilote U n 8 mars ensemble, rapport d'évaluation $19832 \mathrm{~cm}$.

2. Constitué en 1988 afin de faciliter l'accès des Francoontariennes aux études supérieures, le Collectif des femmes francophones du Nord-Est ontarien est à l'origine de plusieurs projets touchant directement ou indirectement la santé. $\mathrm{C}$ e $\mathrm{C}$ ollectif regroupe des représentantes de plusieurs associations féminines, d'établissements d'enseignement secondaires et post-secondaires du nord de I'O ntario, divers regroupements de défense des francophones ainsi que des membres individuelles. L'objectif principal de ce groupe est «d'amener les femmes à se concerter entre elles en matière d'éducation post-secondaire et à se mobiliser autour de cette question» (A dam 1990b:29).

3. Les cinq ateliers-rencontres de trois heures et demie chacun ont comme thèmes: la pré-ménopause et la ménopause, I'influence de l'éducation sexiste sur la santé des femmes et la sexualité féminine, la crise d'identité, l'alimentation et l'éducation physique de la femme de 35 ans et plus, les traitements hormonaux et chirurgicaux (Côté, 1986:106).

4. Ce programme est d'une durée de 15 heures et comporte 6 ateliers:Stressées... moi!!, compréhension de la nature du stress; À l'écoute de mon corps, identification des manifestations du stress dans mon corps; M on stress... jel'identifie, sources internes et externes du stress; $M$ on stress... je l'apprivoise, stratégies personnelles pour faire face au stress; M on stress je l'organise, gestion du temps; M on stress... j'y fait face. 
5. Cette reconnaissance de la spécificité de la santé des femmes francophones n'a pas eu sa place dans le débat public auprès des associations franco-ontariennes ni auprès de tous les intervenants et intervenantes. A insi, dans l'étude sur la présence de professionnels et professionnelles francophones danslesservices de santé et les services sociaux en 0 ntario, publié par le Conseil de planification sociale d'O ttawa- $\mathrm{C}$ arleton, rien ne touche la santé des femmes francophones. Elles sont ignorées tout simplement.

6. Souligné par nous. Dans les documents que nous avons lu pour la période de 1977 à août 1995, c'est la première fois que dansun document public, lesfemmes francophones revendiquent des services en anglais.

7. Ces recommandations ne sont pas sans rappeler celles du Conseil consultatif de l'O ntario sur la condition féminine au sujet de la santé mentale desfemmesen 0 ntario (CCOCF, 1991). En effet, dans toutes les régions, les 0 ntariennes ont reconnu la nécessité de sensibiliser les professionnels et professionnelles de la santé, le personnel judiciaire, de même que le personnel enseignant aux questions de santé des femmes.

\section{Bibliographie}

ACTION ÉDUCATION FEMMES ONTARIO (1993). D ialogue sur les faceurs déterminants en matière de santé au sein des collectivités francophones de l'O ntario. $R$ apport préparé par A cion É ducation F emmes $O$ ntario et soumis au C onseil du premier M inistre sur la santé, le bien-être et la justice sodale, $O$ ttawa, AÉF O ntario, par Geneviève $R$ ail et Suzanne St-Pierre.

ADAM , D yane (1992a). «U n partenariat pour le meilleur ou pour le pire», première partie, F emmes d'action, vol. 21, no 2, 13-16.

ADAM , D yane (1992b). «U n partenariat pour le meilleur ou pour le pire», deuxième partie, F emmes d'action, vol. 21, no 3, 7-10.

ADAM , D yane (1990a). Le stress, c'est... euh... euh... ?, Femmes d'action, vol. 19, no 4, 15-16.

ADAM, Dyane (1990b). «Le Collectif s'en charge: ouvrir les portes du post-secondaire aux femmess, Femmes d'action, vol. 20, no 1, 29-30.

ADAM , Dyane (1989a). «D es corsets aux régimes amaigrissants», Femmes d'adion, vol. 18, no 5, 10-12.

A DAM , D yane (1989b). «Stress au féminin. En $O$ ntario français, un programme adapté aux besoins des femmes en milieu rural», Femmes d'action, vol. 18, no 4, 6-7.

ADAM, Dyane (1989c). «Saines d'esprit, malgré tout. $R$ egard sur notre santé mentale: résumé d'une conférence», Femmes d'adion, vol. 19, no 1, 11-15. 
ASSO CIATIO N DES FER M IĖR ES DE LA PROVIN CE DE L'O NTAR IO (AFO) (1977). R apport du congrès 1977, Fonds FFCF C 53\19\11, Administration nationale, membres et organismes affiliés, A ssociation des fermières de l'O ntario, 1974-1979.

BEAU R EGAR D, M icheline et M aria DE KON IN CK (1991). «Savoirs occultés, soins ignorés, institutions à redéfinir: un programme de recherches féministes en santé desfemmes», $R$ echerches féministes, vol. 4, no 1, 1-10.

BR EEN, M ary J. (1993). M a santé, je m'en occupe! G uide-santé pour les femmes, Ottawa, UCFO, traduction Antoinette Gagné.

BO IVIN , Louise (1983). «R egards sur I'avenir: U n colloque national avec une histoire», Femmes d'adion, vol. 13, no 3, 3-4.

BO UTIN ,T hérèse (1986). «U ne coalition des femmes francophones de l'O ntario: La représentation collective», F emmes d'adion, septembre - octobre - novembre, p. 8.

BR ASSAR D, C laudette (1992). «A uto-santé, médecine douce», dans M arie-Luce G arceau éd. R ele vons le déf! ! A đes du colloque sur l'intervention féministe dans le N ord-est de l'O ntario, 0 ttawa, 151-155.

BRU NET, Lucie (1994). « La maternité et la famille tranforormée par les nouvelles techniques de procréation», Femmes d'action, vol. 23, no 3, 19-22.

BRUNET, Lucie, Chantal P. CHOLETTE et M icheline PICHÉ (1988). «Des préoccupations paroissiales aux préoccupations féministess, Femmes d'action, vol. 17, no 4, 17-18.

CAR DIN AL, Linda (1992). « La recherche sur les femmes francophones vivant en milieu minoritaire: un questionnement sur le féminisme», R echerches féministes, vol. 5, no 1, 5-29.

CAR DIN AL, Linda et C écile CO DER R E (1990). «R econnaître une histoire, le mouvement des femmes francophones hors Q uébec», F emmes d'adion, vol. 19, no 3, 15-17 et 18.

CAR DIN AL, Linda (1992). «T héoriser la double spécificité des Franco-O ntariennes», dans M arieLuce Garceau éd. R elevons le défi! A ctes du colloque sur I'intervention féministe dans le $N$ ord-E st de I'O ntario, 0 ttawa, 177-188.

CAR DIN AL, Linda et Cécile CODER RE (1991). «U ne formation par et pour les femmes. Enquête sur les activités de formation des femmes francophones hors Q uébec», Pour les femmes: éducation et autonomie, rapport no. 4, O ttawa, R N AÉF.

CAR R IÈR E, Fernand (1993). «La métamorphose de la communauté franco-ontarienne, 19601985, dans Cornelius J. Jaenen, éd. Les F ranco-0 ntariens, $O$ ttawa, PU O, 305-340.

CENTR E D'ACCÈS PO U R FEM MES (sd). A utonomel A utonome! A utonome! , dépliant d'information.

COLLOQUE PROVIN CIAL SUR LESAGR ESSION S À CAR ACTÈR E SEX U EL (1994). L es ades du colloque «Sensibiliser, déder, agir», 14,15 et 16 odobre 1994.

COLLECTIF DES FEMMES FR AN COPHONES DU NORD-EST DE L'ONTARIO (sd). $M$ odèles-à-imiter, trousse d'information.

COMITÉ DES SERVICES EN FR AN ÇAIS DU CON SEIL DE PLAN IFICATION SO CIALE D 'O TTAWA-CAR LET ON (1985). Les professionnels francophones dans les services de santé et les services sociaux en 0 ntario: $R$ apport de situation, $O$ ttawa, $O$ ntario, $C$ onseil de planification sociale d'O ttawa Carleton.

Communiqué (1992). vol. 9, no 1.

Communiqué (1987). vol. 4, no 1. 
CONDITIO N FÉM IN IN E CAN ADA (1989). Les dimensions de l'égalité: Suite donnée au plan d'action du gouvernement fédéral concernant les femmes, $O$ ttawa, $M$ inistère des approvisionnements et service.

CONSEIL CONSULTATIF DE L'ONTARIO SUR LA CONDITION FÉM IN INE (1991a). Un document-débat sur les femmes et le vieillissement, Toronto, C CO CF.

CONSEIL C O N SU LTATIF DE L'ON TAR IO SU R LA CONDITIO N FÉM IN IN E (1991b). La santé mentale des femmes en 0 ntario. $R$ eaueil de recommandations présentées au gouvernement de I' 0 ntario, Toronto, CCOCF.

CON SEIL CON SU LTATIF DE L'ONTAR IO SUR LA CON DITION FÉMIN INE (1990). LeS femmes et la santé mentale en 0 ntario, Toronto, C COCF.

CONSEIL DU PREMIER MINISTRE SUR LA SANTÉ, LE BIEN-ÊTRE SOCIAL ET LA JU STICE SO CIALE (1994). La santé pour tous les 0 ntariens. D ialogue provincial sur les facteurs déterminants de la santé,Toronto, Imprimeur de la R eine pour I'O ntario.

CONSEIL DU PREMIER MINISTRE SUR LA SANTÉ, LE BIEN-ÊTRE ET LA JUSTICE SO CIALE (1992). L'enquête sur la santé en 0 ntario 1990. R apport sur les faits saillants, Toronto, $M$ inistère de la santé de l'O ntario.

CO N SEIL DES AFFAIR ES FR AN C O -O N TAR IEN N ES (1985). D onnées générales sur la femme francophone en 0 ntario, Toronto, CAFO.

CO N SEIL DESAFFAIR ES FR AN C O - O N TAR IEN N ES (1986). Symposium pour la femme francophone tenu à Toronto les 25 et 26 odobre, Toronto, CAFO.

CÔTÉ, Éthel (1986). Plus qu'hier, moins que demain, U CFO 1986, 0 ttawa, U CFO.

CR ESSO N, Geneviève (1991). «a santé, production invisible des femmes», R echerches féministes, vol. 4, no 1, 31-44.

DRO U IN , Francine (1981). «Les résolutions... un mal pour un bien!», L e nouveau tablier déposé, no spécial, 78.

DELAGR AVE, Paule (1985). «Le pouvoir médical repose sur la dépendance des patientes», Femmes d'action, vol. 15, no 1.

DU M ON T, M icheline (1986). L e mouvement des femmes hier et aujourd' hui, O ttawa, ICR EF/CR IAW.

ENTR AIDE NORD/SELF-HELP NORTH/W EEDOOKAAZOQIN GEEWEDIN (1989), $\mathrm{G}$ uide des groupes d'entraide de Sudbury-M anitoulin/ $\mathrm{H}$ andbook of Self-H elp/Support $\mathrm{G}$ roup, dans Dyane A dam éd., Sudbury, Entrai de N ord/Self-H elp N orth/Weedookaazoqin G eewedin.

FÉDÉR ATION DES FEM MES CAN ADIEN N ES-FR AN ÇAISES (1981). Femme et francophone: double infériorité, $O$ ttawa, FFCF, par Pauline Proulx.

FÉDÉR ATION DES FEM MES CAN ADIEN N ES-FR AN ÇAISES (1979). La part des femmes, il faut la dire, O ttawa, FFCF.

FÉdÉR ATION DES FEM M ES CAxN ADIEN NES-FR AN ÇAISES DE L'ONTARIO (1992). $C$ essons de penser que l'amour va tout vaincre $L$ a situation des femmes francophones de 45 à 64 ans qui vivent en 0 ntario. R apport final, Sudbury, FFCF $O$ ntario, par M arie- Luce Garceau.

FÉDÉR ATION DES FEM MES CAN ADIEN NES-FR AN ÇAISES, SECTION OTTAWAO U EST (1979). R apport d' activités,FondsFFCF C 53\ 52\ 4 A ctivités Projet $O$ ttawa- $O$ uest, 1979, 1 pièce. Fonds FFC F C 53\47\5 A divités: femmes dans les provinces canadiennes, 0 ntario, 0 riginaux, copies et imprimés, sd, 1976-1981, 1cm. 
GAGN É, Antoinette (1993). «La santé mentale: pas rien qu'une affaire de tête!», C ommuniqué, vol. 10, no 1, 1820.

GAR CEAU, M arie-Luce (1992). D ossier. Q ui sont les F ranco-0 ntariennes, Sudbury, É cole de service social, $U$ niversité Laurentienne, recherche réalisée par Lise $N$ olet et $M$ ireille Leclaire.

GAR CEAU, M arie-Luce (éd.) (1992b). R elevons le défi!. A des du colloque sur l'intervention féministe dans le $\mathrm{N}$ ord-E st de l' $\mathrm{O}$ ntario, $\mathrm{O}$ ttawa, pour le Collectif des femmes francophones du N ord-Est ontarien.

GAU THIER , Lorraine, (1992). «Les nouvelles technologies de la reproduction: un tournant dans «ancien chemin», dans M arie-L uce G arceau éd. R elevons le défi! A des du colloque sur I'intervention féministe dans le $\mathrm{N}$ ord-E st de l'O ntario, 157-174.

GO DIN , Colette (1990). «Le Sud en action», Femmes d'adion, vol. 19, no 3, p. 30.

GRÉGO IR E, N athalie (1993). «Si M élanie avait su!», Femmes d'action, vol. 22, no 3, 31-32.

GROUPE DE TRAVAIL FÉDÉR AL, PROVIN CIAL ET TERRITORIAL SUR LA SANTÉ DES FEM M ES (1990). U n effort conjoint pour la santé des femmes: Plan d'ensemble pour l'élaboration de politiques et programmes, O ttawa, GTFPTSF, avec l'aide de Anne R ochon Ford.

H U OT, Lyse (1981) «Et maintenant, que faisons-nous?», L e nouveau tablier déposé, no spécial, 8-9.

LAM O U R EU X, Jocelyne et Frédéric LESEM AN (1989). «'interface entre les services sociaux publics et les ressources communautaires au Q uébec», Sciences sodales et santé, vol. 7, no 4, 49-67.

LAU R EN CIN , Janine (sd). «La consommation différentielle entre les femmes et les hommes, compte rendu d'une conférence de Colette Guillaumin», Le tablier déposé, vol. 3, no 2, 17-18.

M ESSIER , D enise (1992). «M ourir pour se libérer», dans M arie-Luce G arceau, éd. R elevons le défi!. A ctes du colloque sur l'intervention féministe dans le N ord-E st de I'O ntario, 0 ttawa, 139-149.

PAQ U ETTE C armen et Gracia LALAN DE (1990). «U n féminisme bien vivant: dans l'Est ontarien», Femmes d'adion, vol. 19, no 3, p. 29.

PICHÉ, M icheline (1988). «Être du voyage: regard sur la politisation des groupes de femmes en milieu minoritaire», Femmes d'action, vol. 17, no 4, 14 et 32.

SA ILLAN T, Francine (1991). «Les soins en péril: entre la nécessité et l'exclusion», R echerches féministes, vol. 4, no 1, 11-29.

SA ILLAN T, Francine (1985). «Le mouvement pour la santé des femmes», dans Jacques D ufresne, Fernand D umont etY ves $M$ artin, éd., Traité d' anthropologie médicale L'institution de la santé et de la maladie, 743-62.

SAIN T-PIER R E, Chantal (1992). «D e la fabrication d'une femme épuisée», dans M arie-Luce Garceau, éd. R elevons le défi!. A des du colloque sur l'intervention féministe dans le N ord-E st de l' 0 ntario, 117-137.

SCAPIN ELLO, M ariette (1990). «P remiers pas contre l'isolement», F emmes d'adion, vol. 19, no 3, 28.

THAU VET TE, Patricia (1984). «U CFO : Évolution en survol: 1937-1984», A đion, éducation, femmes, automne, 13-16.

U N IO N DES FER M IÈR ES DE L'O N TAR IO (1987). L'A ssodation desfermières del'O ntario (19371987): Un avenir pour le passé.

U N IO N CU LT U R ELLES DES FR A N C O - O N TAR IEN N ES (U CFO ) (1994). L a santé apprivoisée. $R$ apport final, $O$ ttawa, U CFO. 
UN ION CULTUR ELLE DES FR AN C O-ONTAR IEN N ES (sd). Pleins feux sur la vie. Programme sur la retraite/ et préparation à la retraite, brochure publié à U CFO.

U N 8 M AR S EN SEM BLE (1983a). R apport d'évaluation, Fonds FFCF C 53\52 6 A ctivités- projets, Projet pilote. U n 8 mars ensemble rapport d'évaluation 1983, $2 \mathrm{~cm}$.

UN 8 MAR S EN SEM BLE (1983b). Projet, Pour un 8 mars ensemble, Fonds FFCF C 53\52 15, Activités- projets, Projet pour une 8 mars ensemble, 12 mars 1983, $1 \mathrm{~cm}$.

W ELCH, D avid (1995). «Les Franco- O ntariens: la résistance comme mode de vie», R eflets. R evue ontaroise d'intervention sociale et communautaire, vol. 1, no 1, 20-42. 